\title{
Effect of contrail overlap on radiative impact attributable to aviation contrails
}

Inés Sanz-Morère ${ }^{1}$, Sebastian D. Eastham ${ }^{1}$, Florian Allroggen ${ }^{1}$, Raymond L. Speth ${ }^{1}$, Steven R. H.

5 Barrett $^{1}$

${ }^{1}$ Laboratory for Aviation and the Environment, Massachusetts Institute of Technology, Cambridge, MA 02139, United States of America

10 Corresponding author: Sebastian Eastham (seastham@mit.edu)

\section{Supplementary Information}
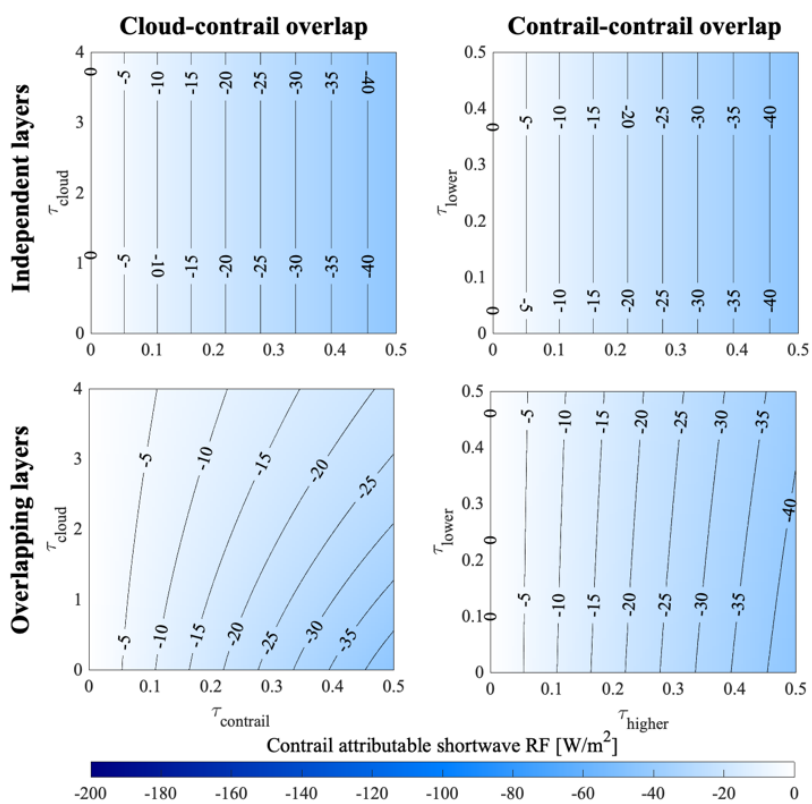

Figure S1. Effect of overlaps on contrail-attributable RFsw as a function of the optical depth $\tau$ of each layer. Left: system contrail-contrail; 15 right: system cloud-contrail. Negative RF is shown in blue and positive RF is shown in red. Lower and upper contrail properties are the following: asymmetry parameter of 0.77 , temperature of $220 \mathrm{~K}$ and $215 \mathrm{~K}$ respectively. Cloud properties are the following: asymmetry parameter of 0.85 , temperature of $260 \mathrm{~K}$. The solar zenith angle $(\theta)$ is held at $45^{\circ}$ for all cases. 

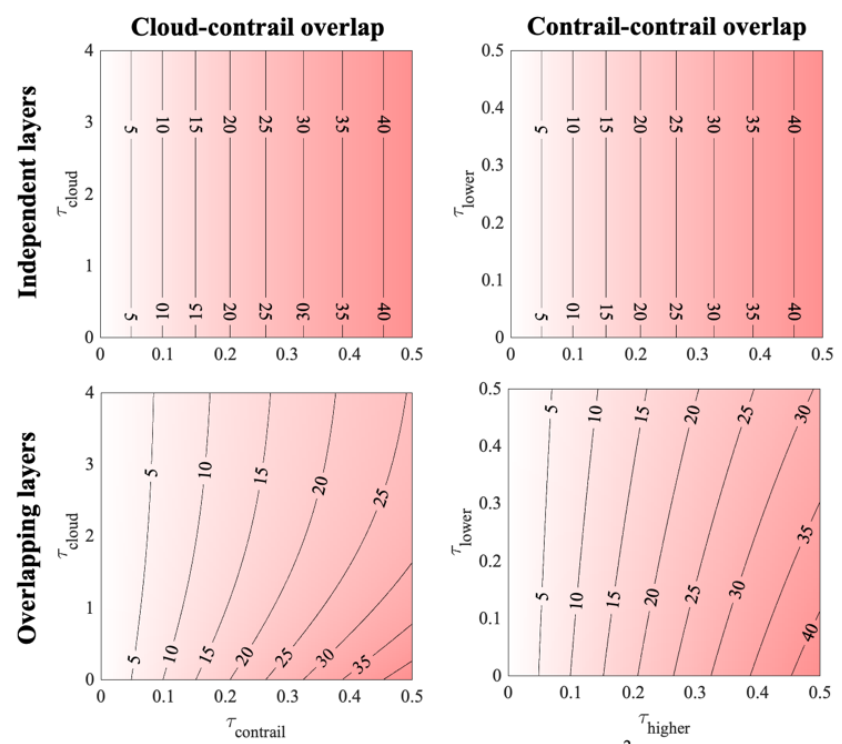

Contrail attributable longwave $\mathrm{RF}\left[\mathrm{W} / \mathrm{m}^{2}\right]$

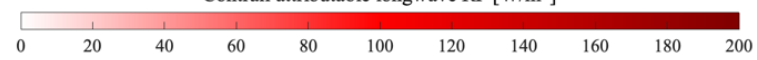

Figure S2. Effect of overlaps on system $R_{L W}$ varying with optical depth $\tau$. Same properties as Fig. S1.
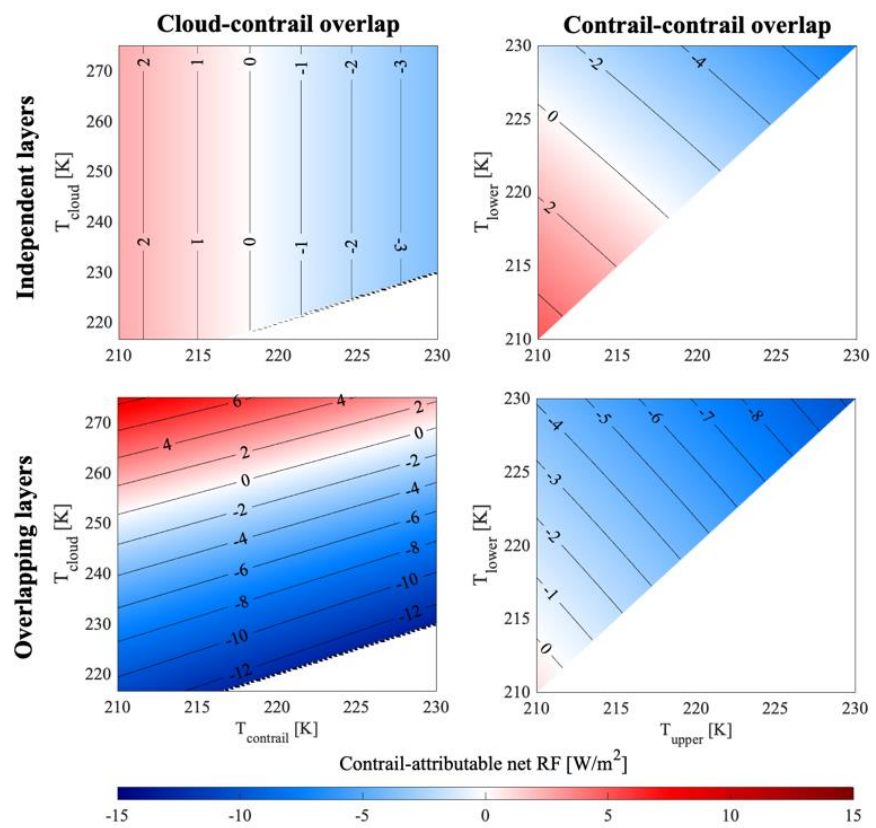

Figure S3. Effect of overlaps on system net RF varying with layer temperature. Left: system contrail-contrail; right: system cloud-contrail. Negative RF is shown in blue and positive RF is shown in red. Lower and upper contrail properties are the following: asymmetry parameter of 0.77 , optical depth of 0.3 . Cloud properties are the following: asymmetry parameter of 0.85 , optical depth of 3 . Fixed $\theta=45^{\circ}$. Cases where the upper layer is warmer than the lower are not shown. 

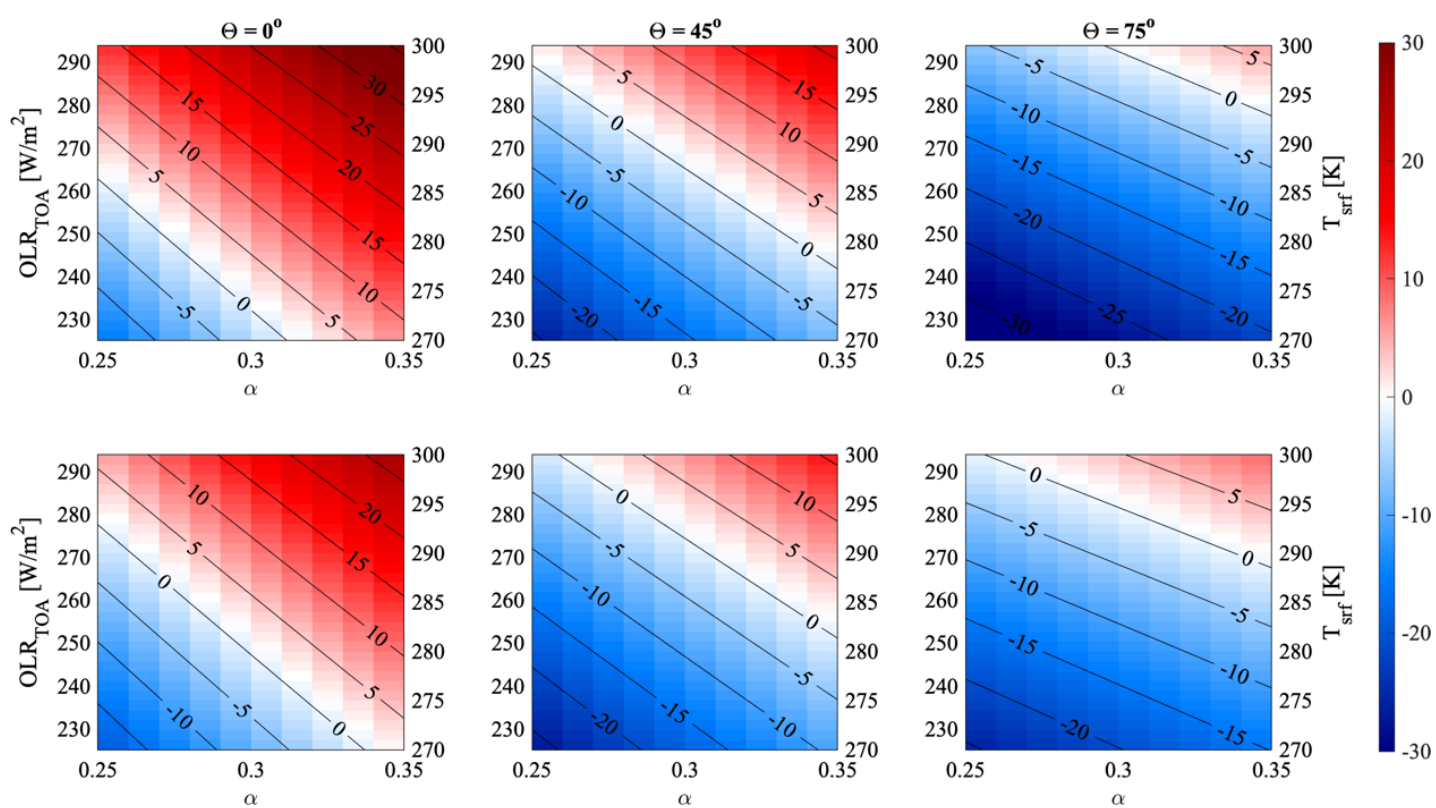

Figure S4. System contrail-contrail net RF in $\mathrm{W} / \mathrm{m}^{2}$, varying with local conditions (solar zenith angle $\theta$ increasing from left to right, outgoing longwave radiation and Earth surface temperature $T_{s r f}$ (based on Corti and Peter, 2009) and albedo $\alpha$ ). Upper row: system RF when contrails considered independent. Lower row: system RF when accounting for total overlap. Negative RF is shown in blue and positive RF is shown in red. Lower and upper contrail properties are the following: asymmetry parameter of 0.77 , optical depth of 0.3 , temperature of $220 \mathrm{~K}$ and

$30215 \mathrm{~K}$ respectively.
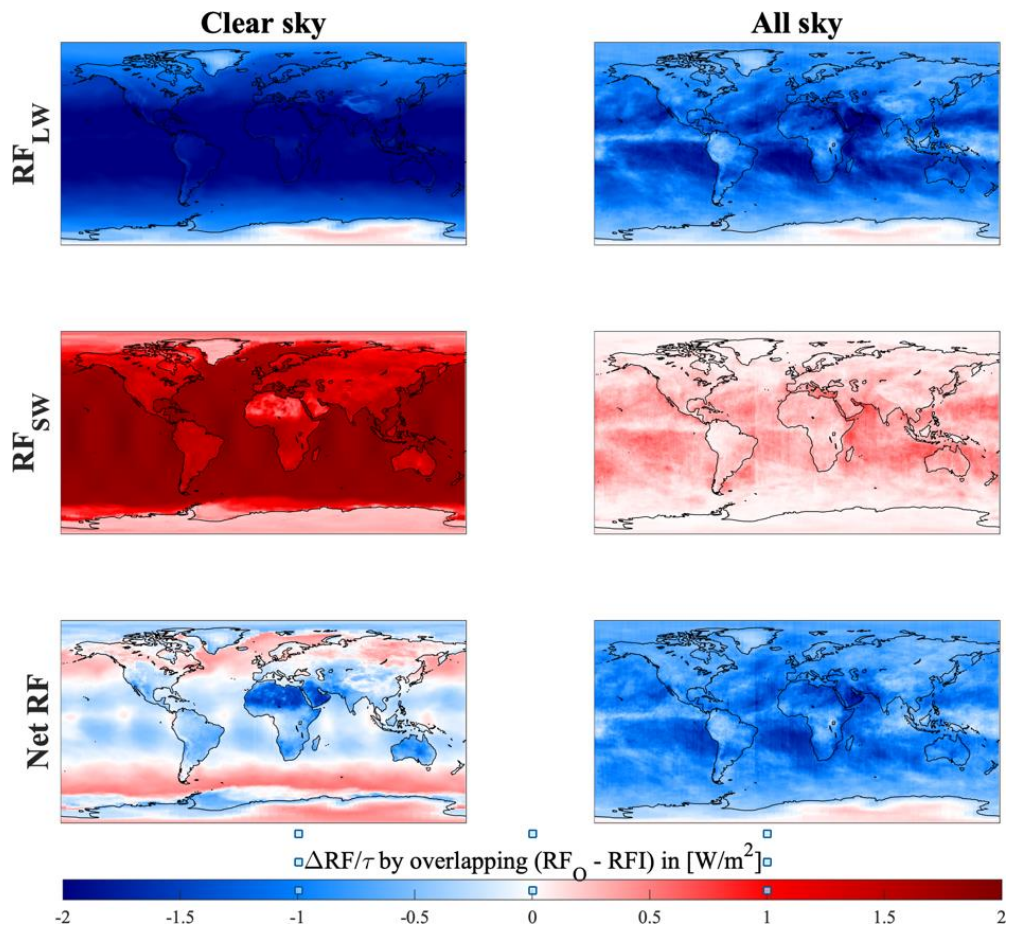

Figure S5. Global sensitivity to contrail-contrail overlap $\left(R F=R F_{O}-R F_{I}\right)$ 